\title{
Recent Nanoarchitectures in Metal Nanoparticle-Graphene Nanocomposite Modified Electrodes for Electroanalysis
}

\author{
Munetaka Oyama, ${ }^{* \dagger}$ Xiaomei Chen, ${ }^{*} * *$ and Xi ChEN*** \\ *Department of Material Chemistry, Graduate School of Engineering, Kyoto University, Nishikyo, \\ Kyoto 615-8520, Japan \\ **College of Biological Engineering, Jimei University, Xiamen 361021, P. R. China \\ *** State Key Laboratory of Marine Environmental Science and Department of Chemistry and the MOE Key \\ Laboratory of Spectrochemical Analysis \& Instrumentation, Xiamen University, Xiamen 361005, \\ P. R. China
}

\begin{abstract}
While increasing attention has been devoted to the use of carbon-based nanomaterials or metal nanoparticles (MNPs) as electrode modifiers for electroanalysis, there is a noticeable development in studies using MNP-graphene nanocomposites or nanohybrids in very recent years. In this review, first, very recent nanoarchitectures in MNP-graphene nanocomposites for modifying electrodes (mainly in 2013) are summarized together with the targets and achievements of electroanalysis. The variety of nanoarchitectures comes from the fact that graphene oxide and metal precursor ions can be reduced chemically or electrochemically, and concurrently or stepwisely. By browsing various preparation methods of the modified electrodes, some characteristic and interesting features of the preparations of MNP-graphene nanocomposites are described together with the possibilities and prospects as electrode modifiers for electroanalysis.
\end{abstract}

Keywords Graphene, graphene oxide, metal nanoparticles, nanocomposites, modified electrodes, electroanalysis, voltammetry, amperometry

(Received March 14, 2014; Accepted March 27, 2014; Published May 10, 2014)

1 Introduction

2 Basic Background

3 Electrochemical Preparations

3.1 Electrochemical reduction of GO followed by electrochemical depositions of MNPs

$3 \cdot 2$ Co-electrodeposition of ERGO and MNPs

3.3 Other electrochemical-based approaches

4 Chemical Preparations

$4 \cdot 1$ Chemical co-reduction of GO and metal ions

$4 \cdot 2$ Chemical reduction of graphene oxide followed by the in situ formation of MNPs

4.3 Chemical reduction of graphene oxide followed by the attachment of MNPs

$\begin{array}{lll}529 & 4 \cdot 4 \text { Other approaches } & \\ 530 & 5 \text { Characteristic Features in the Preparations } & \\ 531 & \text { of MNP-RGO } & 535 \\ & 5 \cdot 1 \text { Remarkable variety of preparation methods } & \\ 5 \cdot 2 \text { GO as the starting material } & \\ & 5 \cdot 3 \text { Various degree of the reduction of GO } \\ & 5 \cdot 4 \text { Functions of GO in chemical reactions } \\ & \quad \text { involving metal ions } \\ 532 & 5 \cdot 5 \text { Variety of graphene derivatives } \\ & 6 \text { Concluding Remarks } \\ & 7 \text { Acknowledgements } \\ & 8 \text { References } & 537 \\ & \end{array}$

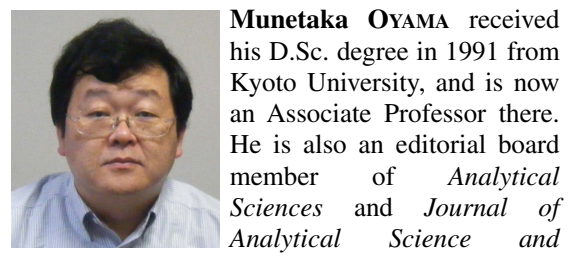

Technology. His current research interests include the development of electroanalytical methods using nanomaterials. He has published over 200 papers in scientific journals.

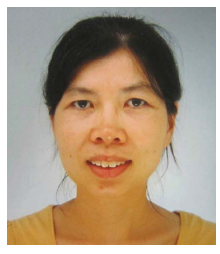

Xiaomei CHEN received her B.S. and Ph.D. degrees from Xiamen University in 2005 and 2011, respectively. She has been working as an Assistant Professor at Jimei University from 2012. Currently, she is a JSPS postdoctoral fellow in Kyoto University (Nov. 2012 - Oct. 2014). Her scientific interests focus on carbon and metal nanomaterials for electrochemical, electrochemiluminescence and bioanalytical applications.

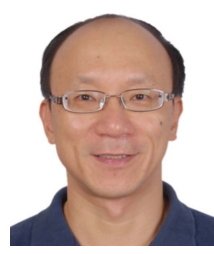

Xi Chen is a Full Professor in the Department of Chemistry at Xiamen University, China He received his $\mathrm{Ph} . \mathrm{D}$. (1996) degree in Analytical Chemistry from Kyoto Institute of Technology, Japan. His research interests cover electrochemical analysis using graphene materials, bio-chemical sensors and pre-extraction materials used in GC/HPLC. He has published over 150 scientific research articles in scientific journals.

$\dagger$ To whom correspondence should be addressed.

E-mail: oyama.munetaka.4m@kyoto-u.ac.jp 


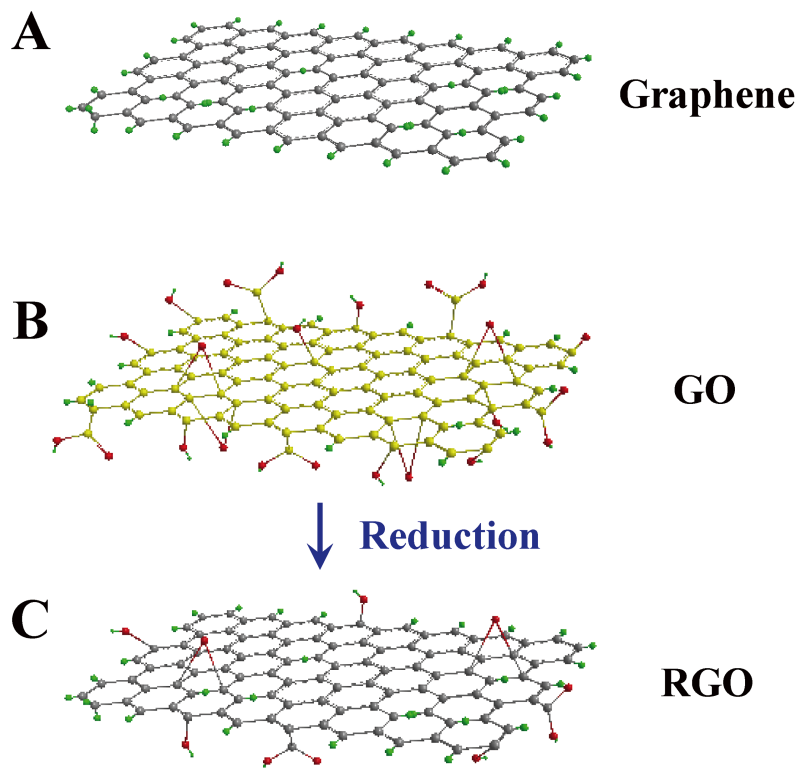

Fig. 1 Structures of (A) graphene, (B) graphene oxide (GO) and (C) reduced graphene oxide (RGO). Red balls indicate oxygen atoms.

\section{Introduction}

In a previous review, one of the authors (M. O.) focused on nanoarchitectures in metal nanoparticle-modified electrodes for electroanalysis, covering studies up to $2009 .{ }^{1}$ While this area is still a focus of active interest, the combination of metal nanoparticles (MNPs) and graphene could be regarded as one of the new emerging hot topics in very recent years. Graphene (whose structure is shown in Fig. 1A) can be utilized as a functional modifier of base electrodes, so it has come to play a very important role in electroanalytical chemistry and analytical sciences as shown in some recent reviews. ${ }^{2-5}$ Guo and Dong summarized the development of graphene and its derivativebased sensing materials for analytical devices for electrochemical and optical sensing. ${ }^{2}$ Chen et al. presented the advances of graphene and graphene-based nanomaterials for electroanalytical chemistry. ${ }^{3}$ Pérez-López and Merkoçi reviewed the trends of carbon nanotubes and graphene in analytical sciences, in particular in biosensing. ${ }^{4}$ Walcalrius et al. reported the trend of nanomaterials for bio-functionalized electrodes, ${ }^{5}$ in which graphene was focused as well as carbon nanotubes and MNPs.

While the combination of MNPs and graphene has been addressed in the above mentioned reviews, there has been an explosion of research in the use of MNP-graphene nanocomposites or nanohybrids. In this field, one typical application is for use as electrocatalysts in fuels cells as reported in some recent papers..$^{6-10}$ However, applications of MNP-graphene nanocomposites as electrode modifiers are quite important also in the field of electroanalysis. Thus, in the present special review, we would like to summarize very recent trends in nanoarchitectures of MNP-graphene nanocomposites for constructing modified electrodes. By reviewing some achievements published mainly in 2013 for electroanalysis, we would like to discuss the possibilities and prospects concerning MNP-graphene nanocomposite modified electrodes.

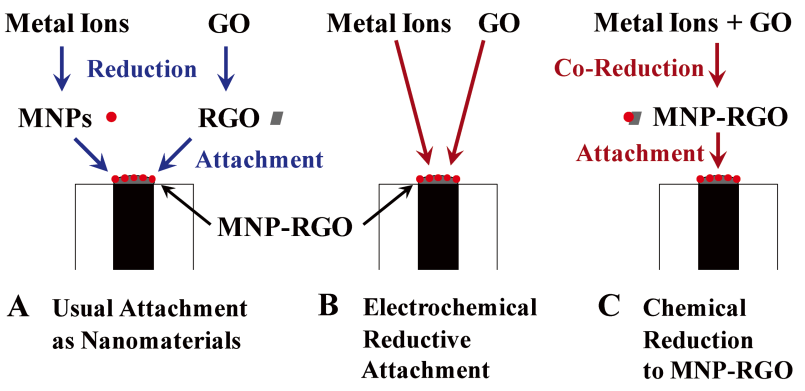

Fig. 2 Schematic representation of the modification of MNP-RGO on the electrode surface. (A) A method to prepare MNPs and RGO separately, and then to attach on the electrode surface. This approach is the most commonly adopted for modifying inert nanomaterials that do not interact with each other. (B) Because metal ions and GO can be reduced electrochemically, reductive attachment on the electrode surface is possible. (C) If chemical co-reduction of metal ions and GO can form MNP-RGO, it can be modified on the electrode surface after the preparation.

\section{Basic Background}

Active research studies on graphene were initiated recently by the first isolation of a single layer graphene sheet with mechanical exfoliation from highly oriented pyrolytic graphite in $2004,{ }^{11}$ and the interesting characteristics and advancements of graphene were summarized later by Geim and Novoselov in 2007. ${ }^{12}$ However, in recent electrochemical works using graphene, the starting material has usually been graphene oxide (GO) that is prepared in a solution phase. Figure 1B shows the typical structure of GO. The preparation method is often referred to as the modified Hummer method. ${ }^{13-15}$ The liquid phase synthesis of GO is suitable for mass-production, and the GO can be chemically reduced to change the electrical characteristics from insulating to conducting. ${ }^{16}$ For example, as a result of the reduction of GO by hydrazine, it was shown that the conductivity of the reduced GO was comparable to that of pristine graphite, but that $\mathrm{O}$ atoms remained in an atomic $\mathrm{C} / \mathrm{O}$ ratio analysis. ${ }^{17}$

In general, it may be difficult to regard reduced GO (RGO) as genuine graphene. Figure $1 \mathrm{C}$ shows a possible state of RGO after the reduction of $\mathrm{GO}$; the graphene structure (Fig. 1A) should be formed after the complete removal of $\mathrm{O}$ atoms. In some literature, RGO or rGO has been used as the abbreviation of "reduced graphene oxide". On the other hand, in some simplified cases, "reduced graphene oxide" has been simply written as "graphene" without a presentation of the results of detailed analysis. Thus, in this review, hereafter we use the abbreviation of "RGO" for "reduced graphene oxide" even if it is differently abbreviated in the original papers, though "graphene" was used in the title of this review to represent the derivatives.

For modifying base electrodes, typically a glassy carbon electrode (GCE), one approach would be a simple modification using MNPs and RGO prepared via the reduction in advance as shown in Fig. 2A. This would be expected from usual modifications of general nanomaterials. However, there is a wide variety in the modification of MNP-graphene nanocomposites (hereafter we abbreviate it as MNP-RGO), because the reduction can be performed using either chemical or electrochemical methods for the precursors of MNPs (i.e., appropriate metal complex ions) and GO, and because the reduction can be carried out concurrently or stepwisely. 
A

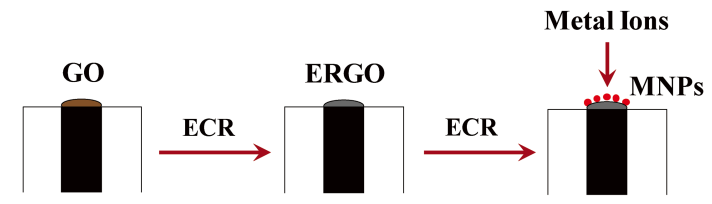

B

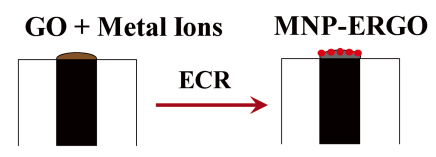

Fig. 3 Schematic representation of electrochemical preparations (A) Electrochemical reduction of GO followed by the electrodeposition of MNPs. (B) Co-electrodeposition of MNPs and ERGO. ECR denotes "electrochemical reduction".

Figures $2 \mathrm{~B}$ and $2 \mathrm{C}$ show the schematic representations of a typical electrochemical reductive attachment and a typical chemical reduction to form MNP-RGO followed by the attachment, respectively. However, in addition to these typical ones, various nanoarchitectures are possible for preparing MNPRGO modified electrodes. In the following sections, we summarize the actual state-of-the-art nanoarchitectures in this field together with the electroanalytical targets.

\section{Electrochemical Preparations}

\subsection{Electrochemical reduction of GO followed by electrochemical depositions of MNPs}

The first example is a stepwise electrochemical preparation, i.e., the first step is the reduction of $\mathrm{GO}$ to form electrochemically reduced GO (ERGO) and the next is the reduction of metal ions to form MNPs on ERGO (Fig. 3A). Ting et al. reported the preparation of gold NPs (AuNPs) decorated ERGO for detecting nitric oxide (NO). ${ }^{18}$ As the actual procedures, GO nanosheets with negative charges were attached on the surface of a GCE via an electrophoretic deposition. Then, the attached GO was electrochemically reduced to form ERGO by cyclic voltammetric scanning. Finally, AuNPs were in situ synthesized onto ERGO film by electrochemically reducing $\mathrm{HAuCl}_{4}$. Because the ERGO network provided high conducting pathways and a large surface area for catalyst support and because AuNPs act as efficient elecrocatalysts for $\mathrm{NO}$ oxidation, the prepared electrode showed good performance for the detection of $\mathrm{NO}$ observing amperometric responses. It was also demonstrated that the electrode could be used to detect the dynamic release of NO from live human umbilical vein endothelial cells (HUVECs).

Concerning the same combination of AuNPs and ERGO, Tang et al. developed a new electrochemical sensor for 4-nitrophenol. ${ }^{19}$ In their experiment, the electrochemical deposition of RGO on a GCE was performed by direct cyclic voltammetric electrolysis under magnetic stirring and $\mathrm{N}_{2}$ bubbling. Then, AuNPs were electrodeposited on ERGO/GCE at a constant potential electrolysis of $\mathrm{HAuCl}_{4}$. It has been reported that the synergetic effect of AuNPs and ERGO as comodifiers greatly facilitate electron-transfer processes, and thus lead to remarkable sensitivity for 4-nitrophenol detection in two detection modes, differential pulse voltammetry and square wave voltammetry. In the paper, the analytical performances for 4-nitrophenol were compared with 12 different modified electrodes in a table. Also, the application for river water samples was described.
As an example of different MNPs by the same scheme of Fig. 3A, Zhang et al. fabricated PdNP-ERGO nanocomposite modified GCE for the detection of nitrite $\left(\mathrm{NO}_{2}{ }^{-}\right){ }^{20}$ In their experiment, firstly, GO modified GCE was prepared by drop casting GO suspensions onto the surface of GCE. Then, GO was reduced by a cyclic voltammetric reduction. PdNPs were electrochemically deposited on the ERGO/GCE by applying a constant potential to reduce $\mathrm{PdCl}_{4}{ }^{2-}$. The prepared electrode exhibited enhanced electrocatalytic behavior to the oxidation of nitrite.

Similarly, the electrodeposition of PdNPs was performed by Hossain and Park. ${ }^{21}$ In their work, chemically modified GO (CGO) was prepared, and then CGO was attached on a gold electrode (AuE) by a cast method. The CGO on the Au electrode was electrochemically reduced by cyclic scans while noting the conductivity change of $\mathrm{CGO}^{22}$ and then PdNPs were electrodeposited on the ERCGO/AuE. The formed electrode was used for the non-enzymatic detection of $\mathrm{H}_{2} \mathrm{O}_{2}$ and glucose by recording amperometric responses. In their paper, the researchers compared the analytical performance for $\mathrm{H}_{2} \mathrm{O}_{2}$ with the previous results obtained using four different modified electrodes.

For preparing ERGO, Shang et al. mixed 1-(2-hydroxyethyl)3-methylimidazolium bis(trifluoromethanesulfonyl) imide dissolved in DMF with a GO aqueous suspension to form an ionic liquid-GO (IL-GO). ${ }^{23}$ It was transferred onto a GCE and electrochemically reduced to form an IL functionalized ERGO. Then, the electrochemical reduction was performed in a solution containing $\mathrm{PdCl}_{2}$ and $\mathrm{HAuCl}_{4}$ to form Pd-Au alloy NPs. The formed electrode was used for the detection of oxalic acid. In the paper, the analytical performances for oxalic acid were compared with the previous results obtained using four different modified electrodes.

As the base electrode, a carbon fiber electrode (CFE) was examined for layer-by-layer assembly of RGO and AuNPs by Du et al. ${ }^{24}$ In the study, referring to a previous preparation of RGO wrapped carbon cloth electrode, ${ }^{25} \quad 0.5 \mathrm{mg} / \mathrm{mL} \quad \mathrm{GO}$ dispersed aqueous solution was prepared, and it was drop-cast onto a CFE. After drying in air, a constant potential electrochemical reduction was performed at first, and then AuNPs were electrochemically deposited from $\mathrm{AuCl}_{4}{ }^{-}$. In addition to AuNPs/ERGO/CFE, the researchers prepared $\mathrm{RGO} / \mathrm{AuNPs} / \mathrm{ERGO} / \mathrm{CFE}$ by repeating one reduction process of GO. The RGO/AuNPs/ERGO/CFE was reported to have good performance for simultaneous determination of uric acid (UA) and dopamine (DA) in comparison with the ERGO/CFE and the AuNPs/ERGO/CFE. ${ }^{24}$

\subsection{Co-electrodeposition of ERGO and MNPs}

While several examples of stepwise preparation (Fig. 3A) are summarized in section $3 \cdot 1$, co-electrodeposition from GO and metal ions can be performed to form MNP-ERGO nanocomposites as shown in Fig. 3B.

Liu et al. reported the formation of an AuNPs-ERGO composite film by cyclic voltammetric electrodeposition on a GCE. ${ }^{26}$ In their experiment, the GO purchased from a provider was exfoliated in $0.1 \mathrm{M}$ carbonate buffer solution by ultrasonication, and then, the cyclic voltammetric electrodeposition was performed for the dispersion containing $\mathrm{GO}$ and $\mathrm{HAuCl}_{4}$. An electrochemical quartz crystal microbalance was used to trace the film formation processes and detect the interactions of arsenic species with the AuNP-ERGO film. The formed film was used in anodic stripping voltammetry analysis of As(III). In their paper, the researchers compared the analytical performance of As(III) with 
the previous results obtained using 11 different modified electrodes.

$\mathrm{Lu}$ et al. also used the co-electrodeposition method. ${ }^{27}$ However, as a difference from Ref. 26, they added chitosan, which has excellent film-forming ability and good water permeability, into $0.1 \mathrm{M}$ acetate buffer suspension containing $\mathrm{HAuCl}_{4}$ and $\mathrm{GO}$, followed by the cyclic voltammetric electrodeposition. The formed film was used in differential pulse anodic stripping voltammetry analysis of $\mathrm{Pb}(\mathrm{II})$. In the paper, the analytical performances for $\mathrm{Pb}$ (II) were compared with the previous results obtained using seven different modified electrodes.

For preparing PdNPs, Palanisamy et al. pretreated $\mathrm{PdCl}_{2}$ and $\mathrm{GO}$ before electrolysis. ${ }^{28}$ Actually, the mixture of $\mathrm{GO}$ and $\mathrm{PdCl}_{2}$ was sonicated to attach $\mathrm{Pd}^{2+}$ on to $\mathrm{GO}$ sheets, and then centrifuged to remove free $\mathrm{Pd}^{2+}$. The resulting $\mathrm{Pd}^{2+} / \mathrm{GO}$ solution was drop casted onto a GCE and reduced at a constant potential to form PdNPs-RGO. The analytical performances for DA were explored in this work, and were compared with the previous results obtained using five different modified electrodes.

\subsection{Other electrochemical-based approaches}

Electrochemical methods have been used for reducing GO and metal precursor ions as summarized above. However, in some cases, non-electrochemical processes can be combined with electrochemical processes for unique nanostructuring.

Qin et al. developed in situ synthesis of highly loaded ultrafine PdNP-decorated GO for glucose biosensor applications. ${ }^{29}$ In the study, referring to a previous work on the reaction of a watersoluble $\mathrm{Pd}$ precursor and $\mathrm{GO},{ }^{30}$ a water-insoluble precursor, $\mathrm{Pd}(\mathrm{OAc})_{2}$, was used. By dissolving it in 1,2-dichloroethane and by volatilizing, the contact between $\mathrm{Pd}(\mathrm{OAc})_{2}$ and $\mathrm{GO}$ dispersion was enhanced, and consequently, highly loaded and ultrafine PdNPs supported on GO (PdNP-GO) was synthesized. While the PdNP-GO was obtained via this non-electrochemical manner, it was reduced to PdNP-ERGO after dropping a dispersion of PdNP-GO on a GCE and air drying. In this manner, a PdNP-ERGO based glucose sensor was constructed with the modification of glucose oxidase and applied for amperometric detection. In their paper, the researchers compared the analytical performance of glucose with earlier results obtained using four different MNPs and carbon nanomaterials-modified electrodes.

Instead of the electrochemical reduction of GO, Wang et al. adopted a chemical reduction of GO in their work to enhance the stability of modified electrodes. ${ }^{31} \mathrm{GO}$ was reduced by hydrazine and mixed with chitosan (CS) to form the RGO-CS composite. After dropping a homogeneous solution of RGO-CS on a GCE and air drying, the PdNPs were electrochemically formed on the surface of the modified GCE with cyclic potential scans. The thus fabricated electrode was used for simultaneous determination of ascorbic acid (AA), DA and UA with cyclic voltammetry and differential pulse voltammetry. As the merits of the proposed electrode, stability, sensitivity, facility and economy were mentioned.

\section{Chemical Preparations}

\section{4-1 Chemical co-reduction of GO and metal ions}

As mentioned in the last example in section $3 \cdot 3,{ }^{31} \mathrm{GO}$ can be reduced using chemical reagents, e.g., hydrazine. ${ }^{17}$ Also, MNPs can be prepared via chemical reductions from appropriate metal precursor ions. Thus, chemical methods can be utilized to reduce GO and metal ions concurrently (Fig. 4A).

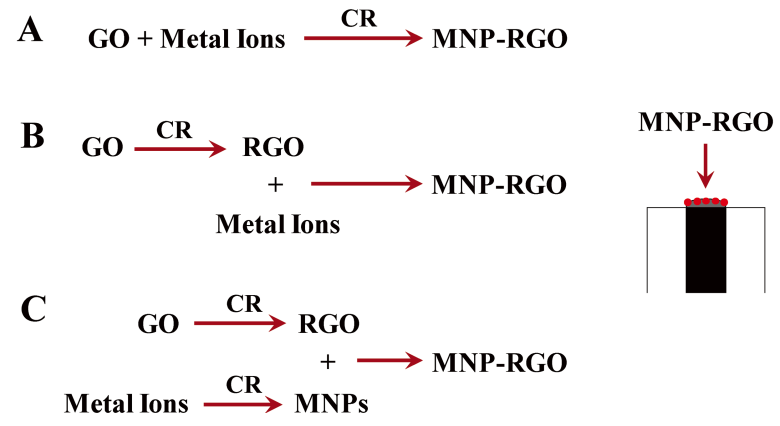

Fig. 4 Schematic representation of chemical preparations. (A) Chemical co-reduction of GO and metal ions. (B) Chemical reduction of GO followed by the in situ formation of MNPs. (C) Chemical reduction of GO followed by the attachment of prepared MNPs. CR denotes "chemical reduction". In all the cases, MNP-RGO was modified on the electrode surface after the preparation of MNP-RGO.

As the first example of this chemical co-reduction of GO and metal ions, Ma et al. reported a chemical co-reduction procedure using $\mathrm{NaBH}_{4}$, in which the reduction of GO and deposition of AuNPs on graphene nanosheets were achieved in a single-step process. ${ }^{32}$ Compared with previous single step approaches,${ }^{33}$ it was mentioned that nearly monodisperse AuNPs uniformly dispersed on the surface of graphene nanosheets without aggregation using the proposed method. The dispersion of AuNPs-RGO was dropped onto the surface of the GCE, followed by drying in an infrared lamp. The fabricated electrode was applied to the simultaneous determination of hydroquinone (HQ) and catechol (CC). In their paper, the researchers compared the analytical performance of $\mathrm{HQ}$ and $\mathrm{CC}$ with the previous results obtained using six different modified electrodes.

As for the preparation of AuNP-RGO via a co-reduction method, the use of sodium citrate was previously reported to be applicable together with heat treatment at $80^{\circ} \mathrm{C} .{ }^{34}$ Cui and Zhang utilized this method for preparing AuNP-RGO, and modified a GCE using the DMF/aqueous (9:1) solution containing the AuNP-RGO. ${ }^{35}$ The performance of the modified electrode was studied for the detection of epinephirine by comparing AuNPs/GCE and RGO/GCE. While sodium citrate was used to prepare AuNP-RGO from $\mathrm{AuCl}_{4}{ }^{-}$and $\mathrm{GO}$, the researchers used hydrazine in the reduction of GO to RGO.

While nanocomposite materials such as AuNP-RGO can be normally prepared in solutions or as solids, one-pot green synthesis of self-assembled AuNP-RGO hybrid membranes was reported by Zhang et al. ${ }^{36}$ They used glucose for reducing GO and $\mathrm{AuCl}_{4}^{-}$by heating at $95^{\circ} \mathrm{C}$, and prepared the hybrid membrane film with polyethylene terephthalate (PET). The hybrid membrane was transferred onto the surface of a GCE, and the sensing ability for $\mathrm{H}_{2} \mathrm{O}_{2}$ was studied.

For preparing a nanocomposite of silver NPs (AgNPs) and RGO, Kaur et al. reported a synthetic method to heat the mixture of $\mathrm{GO}$ and silver nitrate aqueous solution at $80^{\circ} \mathrm{C}$ in the presence of NaOH. ${ }^{37}$ The formed AgNP-RGO was dispersed in an aqueous solution containing Nafion, placed on the GCE, and dried in air. The fabricated electrode exhibited excellent electrocatalytic activity toward the oxidation of AA, DA, UA and tryptophan, allowing for the simultaneous determination of their quaternary mixture. The analysis was applied for the determination of AA and DA in vitamin $\mathrm{C}$ tablets.

Wang et al. reported one pot green synthesis of AgNP-RGO with a hydrothermal method $\left(190^{\circ} \mathrm{C}, 5 \mathrm{~h}\right)$ using gallic acid as a reducing agent. ${ }^{38}$ In this method, AgNPs with an average size of 
$12 \mathrm{~nm}$ were successfully decorated on RGO sheets, and applied as a non-enzymatic $\mathrm{H}_{2} \mathrm{O}_{2}$ sensor by modifying a GCE with AgNP-RGO using Nafion.

Meanwhile, Li et al. reported a green synthesis of AgNP-GO nanocomposite using glucose as a reducing and stabilizing reagent. ${ }^{39}$ Compared with the previous AgNP-GRO, ${ }^{37,38} \mathrm{Ag}^{+}$ was similarly reduced but GO was not reduced, which followed the manner of the previous fabrication of AgNP films on GO. ${ }^{40}$ The thus prepared AgNP-GO nanocomposite was dissolved in DMF, cast on the surface of the GCE, and dried with an infrared lamp. Whereas the target was only tryptophan in this work, the determination performances were compared with the previous results obtained using 21different modified electrodes.

Bimetallic NPs can be prepared by the chemical co-reduction method. Wu et al. reported the preparation of $\mathrm{Cu}-\mathrm{Ag}$ bimetallic NPs on RGO and applied to an amperometric $\mathrm{H}_{2} \mathrm{O}_{2}$ sensor. ${ }^{41}$ In the preparation, ethylenediamine was at first modified on GO to anchor $\mathrm{Cu}^{2+}$ and $\mathrm{Ag}^{+}$, and then the co-reduction was performed using hydrazine. Yan et al. reported simultaneous electrochemical detection of AA, DA and UA using RGO modified with Pd-Pt bimetallic NPs. ${ }^{42}$ In their preparation, poly(diallyldimethylammonium chloride) (DPPA) was used according to a previous report for anchoring $\mathrm{PtCl}_{6}^{2-}$ and $\mathrm{PdCl}_{4}{ }^{2-} .43$ For preparing similar Pd-Pt bimetallic NPs, another anchoring with $\mathrm{Sn}^{2+}$ was also reported. ${ }^{44}$

Chen et al. reported a synthetic method of Pt-Pd bimetallic nanocubes on RGO using $N, N$-dimethylformamide (DMF) as a bifunctional solvent for the reductions of both GO and metal ions and for confining the growth of Pt-Pd nanocubes on the surface. $^{10}$ By using the Pt-Pd bimetallic nanocube-RGO, nonenzymatic sensing of glucose at neutral $\mathrm{pH}$ was explored, and the performances were compared with the previous results obtained using seven different modified electrodes. ${ }^{45}$

\subsection{Chemical reduction of graphene oxide followed by the in situ formation of MNPs}

While GO can be concurrently treated with the reductive formation of MNPs from metal ions, another approach would be the reduction of GO to form RGO (as the first step) followed by the in situ formation of MNPs from metal ions (as the second step) to form MNP-RGO (Fig. 4B). Although the chemical reduction of $\mathrm{GO}$ is the usual technique as mentioned in section $4 \cdot 1$, other methods, e.g., solvent-assisted thermal reduction, are also possible for preparing RGO. ${ }^{46}$

Chen et al. reported a preparation of ultrafine PdNPs on RGO for sensing hydrogen peroxide. ${ }^{47}$ While the preparation of clean well-dispersive ultrafine PdNPs on GO by the redox reaction between $\mathrm{PdCl}_{4}{ }^{2-}$ and GO was reported previously, ${ }^{30}$ in Ref. 47 the thermal reduction of $\mathrm{GO}$ was performed (in an oil bath at $100^{\circ} \mathrm{C}$ for $24 \mathrm{~h}$ ) before reacting with $\mathrm{PdCl}_{4}{ }^{2-}$. As a result, ultrafine, well-dispersed and clean PdNPs were modified on RGO nanosheets with higher conductivity. The PdNP-RGO nanocomposite was fixed on the surface of GCE with $0.5 \%$ Nafion ethanol solution, and applied for electrochemical detection of $\mathrm{H}_{2} \mathrm{O}_{2}$ utilizing the higher performance for the electrocatalytic reduction. In the study, the sensing performances for $\mathrm{H}_{2} \mathrm{O}_{2}$ were compared with the previous results obtained using eight different modified electrodes.

Furthermore, the researchers verified the same approach was applicable to prepare PtNP-RGO nanocomposite, ${ }^{48}$ while it was previously found that $\mathrm{Pt}$ nanoflowers could be formed on GO using a co-reduction method with ethanol at room temperature. ${ }^{49}$ The fabricated PtNP-RGO modified GCE was examined as a non-enzymatic oxalic acid sensor. ${ }^{48}$

In order to prepare RGO from GO, Zhang et al. adopted a treatment of GO with DA. ${ }^{50}$ Because polydopamine (PDA) was prepared by self-polymerization of DA, PDA/RGO was reported to be formed in the treatment of GO and DA previously. ${ }^{51}$ After preparing the PDA/RGO, well-dispersed PtNPs were modified on PDA/RGO by reducing $\mathrm{PtCl}_{6}^{2-}$ with $\mathrm{NaBH}_{4}$. The isolated powder of PtNP-PDA/RGO was dispersed in water, cast onto the surface of GCE, and dried in air. The electrocatalytic activities of the thus prepared electrode were examined for the detection of $\mathrm{H}_{2} \mathrm{O}_{2}$ and $\mathrm{O}_{2} .{ }^{50}$

\subsection{Chemical reduction of graphene oxide followed by the attachment of MNPs}

By performing special treatments during the reduction of GO, it is possible to attach MNPs prepared in advance separately (Fig. 4C). As one of the examples, similar to the case of PDA/ $\mathrm{RGO}^{50}$ discussed in section 4.2, Zhu et al. prepared PVP functionalized graphene from GO by using hydrazine for reduction in the presence of PVP. ${ }^{52}$ Then, hollow AuNPs were prepared in accordance with the procedure described in a previous report, ${ }^{53}$ and mixed with the PVP/RGO to attach the hollow AuNPs. The prepared nanocomposite was dispersed in DMF, dropped on the surface of GCE and dried in an infrared lamp. The modified electrode was applied for the selective detection of DA in the presence of AA and UA. ${ }^{52}$

Instead of the simple reduction of $\mathrm{GO}$, it is known that water soluble RGO, or graphene, can be synthesized by introducing a small number of $p$-phenyl- $\mathrm{SO}_{3} \mathrm{H}^{54}$ This sulfonated graphne (SG) was prepared by $\mathrm{Si}$ and Samulski to overcome the aggregations and precipitations of graphene that was yielded by removing the oxygen functionality of GO. ${ }^{54}$ Using this $\mathrm{SG}, \mathrm{Li}$ et al. reported a sensitive and selective nitrite sensor based on the AuNPs-SG modified GCE. ${ }^{55}$ For the fabrication of the modified electrode, SG was modified first by casting and air dried, and then AuNPs prepared in advance were attached by achieving a saturated adsorption by electrostatic attraction. In the work, some analytical performance characteristics for nitrite were compared with the previous results using seven different modified electrodes. The same modified electrode was also applied for the simultaneous determination of HQ and CC. ${ }^{56}$ In the related paper, anti-adsorption properties of AuNP-GS toward aromatic species were noted.

In section $4 \cdot 1$, one example for the preparation of AuNP-RGO hybrid PET membranes was described as Ref. 36. Similar to the PET membranes, some efforts have been devoted to form freestanding paper-like materials composed of RGO by Duan and coworkers. ${ }^{57}$ In their work, the GO paper was fabricated using a mold-casting method. Namely, an aqueous dispersion of GO was placed in a casting mold, and an integrated layer of GO paper with smooth surfaces could be peeled out after water was completely evaporated. A RGO paper was prepared by the chemical reduction of a GO paper with $\mathrm{HI}$, based on procedures described in a previous work. ${ }^{58}$ Following their earlier 2D-assembly of AuNPs on GO paper by a dip casting method, ${ }^{59}$ 2D-assembly of Au@Pt NPs were similarly formed on RGO paper, and the fabricated Au@Pt NPs attached RGO paper electrode was applied as a flexible biosensor to monitor live cell secretion of nitric oxide. ${ }^{60}$

\subsection{Other approaches}

As chemical approaches, the co-reduction of GO and metal ions (Fig. 4A), the in situ formation of MNPs after the formation of RGO (Fig. 4B), and the attachment of MNPs on RGO (Fig. 4C) are the three major processes as summarized above. However, Zhuo et al. reported a different approach via selfcatalysis reduction at room temperature; i.e., MNPs with 
Table 1 Summary of the recent electroanalytical works cited in this review

\begin{tabular}{|c|c|c|c|c|c|c|c|c|c|}
\hline Ref. & $\begin{array}{c}\text { Base } \\
\text { Electrode }^{\mathrm{a}}\end{array}$ & $\begin{array}{l}\text { Metal of } \\
\text { MNPs }\end{array}$ & $\begin{array}{l}\text { Preparation } \\
\text { Method }^{\mathrm{b}}\end{array}$ & Analyte $^{c}$ & $\begin{array}{l}\text { Detection } \\
\text { Method }^{\mathrm{d}}\end{array}$ & Comparison $^{\mathrm{e}}$ & Interference $^{f}$ & Application ${ }^{g}$ & $\begin{array}{l}\text { Submitted } \\
\text { month, year }\end{array}$ \\
\hline 18 & GCE & $\mathrm{Au}$ & 3.1 & NO & A & $8(\mathrm{txt})$ & $\begin{array}{l}\text { Oxalate, glucose } \\
\text { UA, AA, } \mathrm{Na}^{+}, \mathrm{Cl}^{-}\end{array}$ & NO from HUVECs & Mar. 2013 \\
\hline 19 & GCE & $\mathrm{Au}$ & 3.1 & 4-Nitrophenol & DPV & $12(\mathrm{tbl})$ & 9 phenol derivatives & River water (R) & May 2013 \\
\hline 20 & GCE & $\mathrm{Pd}$ & 3.1 & $\mathrm{NO}_{2}^{-}$ & A & - & $\begin{array}{l}\mathrm{Cl}^{-}, \mathrm{ClO}_{4^{-}}, \mathrm{NO}_{3}^{-}, \mathrm{K}^{+}, \\
\mathrm{Ca}_{2}^{+}, \mathrm{SO}_{4^{2-}}\end{array}$ & $\begin{array}{l}\text { Drinking and river } \\
\text { water }(\mathrm{R})\end{array}$ & Feb. 2013 \\
\hline \multirow[t]{2}{*}{21} & $\mathrm{AuE}$ & $\mathrm{Pd}$ & 3.1 & $\mathrm{H}_{2} \mathrm{O}_{2}$ & A & $4(\mathrm{tbl})$ & $\mathrm{AA}, \mathrm{UA}, \mathrm{AP}$ & - & Mar. 2013 \\
\hline & & & & Glucose & A & $2(\mathrm{txt})$ & $\mathrm{AA}, \mathrm{UA}, \mathrm{AP}$ & - & \\
\hline 23 & GCE & $\mathrm{Au}-\mathrm{Pd}$ & 3.1 & Oxalic acid & LSV & $4(\mathrm{txt})$ & - & Spinach & Oct. 2012 \\
\hline 24 & CFE & $\mathrm{Au}$ & 3.1 & $\mathrm{DA}+\mathrm{UA}$ & DPV & $3(\mathrm{txt})$ & $\begin{array}{l}\mathrm{Na}^{+}, \mathrm{K}^{+}, \mathrm{NH}_{4}^{+}, \\
\mathrm{Cl}^{-}, \mathrm{NO}_{3}^{-}, \mathrm{AA},\end{array}$ & - & (May 2013) \\
\hline 26 & GCE & $\mathrm{Au}$ & 3.2 & $\mathrm{As}^{3+}$ & ASV & $11(\mathrm{tbl})$ & $\mathrm{Cu}^{2+}$ & $\begin{array}{l}\text { Tap, spring and river } \\
\text { water }(\mathrm{R})\end{array}$ & Feb. 2013 \\
\hline 27 & GCE & $\mathrm{Au}$ & 3.2 & $\mathrm{~Pb}^{2+}$ & DPASV & $7(\mathrm{tbl})$ & $\begin{array}{l}\mathrm{Cu}^{2+}, \mathrm{Cd}^{2+} \text { and } \\
7 \text { cations }\end{array}$ & River water (R) & Nov. 2012 \\
\hline 28 & GCE & $\mathrm{Pd}$ & 3.2 & DA & LSV & $5(\mathrm{tbl})$ & AA, UA, glucose & - & Feb. 2013 \\
\hline 29 & GCE & $\mathrm{Pd}$ & 3.3 & Glucose & A & $5(\mathrm{tbl})$ & AA, UA & Human blood serum & Aug. 2012 \\
\hline 31 & GCE & $\mathrm{Pd}$ & 3.3 & $\mathrm{AA}+\mathrm{DA}+\mathrm{UA}$ & DPV & - & - & - & Dec. 2012 \\
\hline 32 & GCE & $\mathrm{Au}$ & 4.1 & $\mathrm{HQ}+\mathrm{CC}$ & DPV & $6(\mathrm{tbl})$ & $\begin{array}{l}\text { AA, UA, phenol, } \\
9 \text { ions, resorcinol }\end{array}$ & Tap water (R) & Sep. 2012 \\
\hline 35 & GCE & $\mathrm{Au}$ & 4.1 & EP & $\mathrm{CV}$ & - & AA & EP solution (R) & Nov. 2011 \\
\hline 36 & none & $\mathrm{Au}$ & 4.1 & $\mathrm{H}_{2} \mathrm{O}_{2}$ & $\mathrm{~A}$ & - & $\begin{array}{l}\text { AA, UA, DA, } \\
\text { glucose }\end{array}$ & - & Sep. 2013 \\
\hline 37 & GCE & $\mathrm{Ag}$ & 4.1 & $\begin{array}{l}\mathrm{AA}+\mathrm{DA}+\mathrm{UA} \\
+ \text { tryptophan }\end{array}$ & LSV & - & $\begin{array}{l}\mathrm{Na}^{+}, \mathrm{K}^{+}, \mathrm{Mg}^{2+}, \mathrm{Zn}^{2+}, \\
\text { glucose }\end{array}$ & $\begin{array}{l}\text { Vitamin } \mathrm{C} \text { tablet and } \\
\text { human urine }(\mathrm{R})\end{array}$ & Jan. 2013 \\
\hline 38 & GCE & $\mathrm{Ag}$ & 4.1 & $\mathrm{H}_{2} \mathrm{O}_{2}$ & A & - & $\begin{array}{l}\text { AA, UA, DA, } \\
\text { glucose }\end{array}$ & - & Apr. 2013 \\
\hline 39 & GCE & $\mathrm{Ag}$ & 4.1 & Tryptophan & DPV & $21(\mathrm{tbl})$ & $\begin{array}{l}\text { AA, UA, DA, } \\
\text { glucose, }\end{array}$ & $\begin{array}{l}\text { Human blood serum } \\
(\mathrm{R})\end{array}$ & Jul. 2012 \\
\hline 41 & GCE & $\mathrm{Ag}-\mathrm{Cu}$ & 4.1 & $\mathrm{H}_{2} \mathrm{O}_{2}$ & A & $2(\mathrm{txt})$ & - & - & Aug. 2013 \\
\hline 42 & GCE & $\mathrm{Pd}-\mathrm{Pt}$ & 4.1 & $\mathrm{AA}+\mathrm{DA}+\mathrm{UA}$ & A, DPV & $6(\mathrm{tbl})$ & $\begin{array}{l}\text { Glucose, citric acid, } \\
\mathrm{Na}^{+}, \mathrm{K}^{+}, \mathrm{Cl}^{-}\end{array}$ & $\begin{array}{l}\text { Human blood serum } \\
\text { and urine }(\mathrm{R})\end{array}$ & Mar. 2013 \\
\hline 45 & GCE & $\mathrm{Pd}-\mathrm{Pt}$ & 4.1 & Glucose & A & 7 (tbl) & $\begin{array}{l}\text { AA, UA, } \mathrm{Cl}^{-} \\
p \text {-acetamidophenol }\end{array}$ & - & Sep. 2013 \\
\hline 47 & GCE & $\mathrm{Pd}$ & 4.2 & $\mathrm{H}_{2} \mathrm{O}_{2}$ & A & $8(\mathrm{tbl})$ & AA, DA, glucose & - & Dec. 2012 \\
\hline 48 & GCE & $\mathrm{Pt}$ & 4.2 & Oxalic acid & DPV & $6(\mathrm{tbl})$ & - & Spinach (R) & Feb. 2013 \\
\hline 50 & GCE & $\mathrm{Pt}$ & 4.2 & $\mathrm{H}_{2} \mathrm{O}_{2}$ & A & $2(\mathrm{txt})$ & $\begin{array}{l}7 \text { ions, AA, UA, } \\
\text { glucose, L-glycine, }\end{array}$ & - & May 2013 \\
\hline 52 & GCE & $\begin{array}{l}\text { hollow- } \\
\mathrm{Au}\end{array}$ & 4.3 & DA & A, DPV & $5(\mathrm{txt})$ & $\mathrm{AA}, \mathrm{UA}$ & DA solution (R) & Feb. 2013 \\
\hline 55 & GCE & $\mathrm{Au}$ & 4.3 & $\mathrm{NO}_{2}^{-}$ & A & $7(\mathrm{tbl})$ & $\begin{array}{l}12 \text { ions, HQ, CC, } \\
\text { aminophenol }\end{array}$ & River water & Jan. 2013 \\
\hline 56 & GCE & $\mathrm{Au}$ & 4.3 & $\mathrm{HQ}+\mathrm{CC}$ & DPV & $5(\mathrm{txt})$ & $\begin{array}{l}\text { Phenol, glucose, } \\
\text { resorcinol, AA }\end{array}$ & Tap water $(\mathrm{R})$ & Jan. 2013 \\
\hline 60 & none & $\mathrm{Au} @ \mathrm{Pt}$ & 4.3 & NO & A, DPV & $5(\mathrm{tbl})$ & $\begin{array}{l}\mathrm{AA}, \mathrm{DA}, \mathrm{UA}, \mathrm{NO}_{2}^{-} \\
\text {and } 17 \text { ions }\end{array}$ & NO from HUVECs & Feb. 2013 \\
\hline 62 & GCE & $\mathrm{Pt}$ & 4.4 & $\mathrm{H}_{2} \mathrm{O}_{2}$ & A & $8(\mathrm{tbl})$ & $\begin{array}{l}\text { AA, AP, glucose, } \\
\text { citric acid, urea }\end{array}$ & - & Jan. 2012 \\
\hline
\end{tabular}

a. GCE; glassy carbon electrode, AuE; gold electrode, CFE; carbon fiber electrode. b. Classification in this review. c. Combination with "+" denotes "simultaneous determination." DA; dopamine, UA; uric acid, AA; ascorbic acid, HQ; hydroquinone, CC; catechol, EP; epinephrine. d. A; amperometry, DPV; differential pulse voltammetry, LSV; linear sweep voltammetry, ASV; anodic stripping voltammetry, DPASV; differential pulse anodic stripping voltammetry. e. Number of other reported modified electrodes with which the electroanalytical performance features were compared in the text (txt) or as a table (tbl) including those in supporting information. f. Representative ions and molecules for which the interferences were examined. AP; acetaminophen. g. (R) means recovery percentages were examined for applying real samples. HUVECs; human umbilical vein endorthelial cells. h. Month and year of publication online in parentheses.

desirable size and morphology were decorated at first on GO, and then used as catalyst to accelerate the hydrolysis reaction of $\mathrm{NaBH}_{4}$ to reduce the GO. ${ }^{61}$ As the advantageous points of this approach, they summarized (i) possible control of the morphology and size of MNPs, (ii) a uniform distribution of MNPs with high density, (iii) no use of toxic reductants, (iv) reaction at room temperature and (v) applicability in a wide range of $\mathrm{pH}$ values.
As a method without using chemical or electrochemical reductions, Zhang et al. reported a microwave-assisted synthesis of PtNP-RGO for applying to a $\mathrm{H}_{2} \mathrm{O}_{2}$ sensor. ${ }^{62}$ Also, photochemical synthesis of AgNPs, PdNPs, AuNPs or PtNPs was reported on $\mathrm{ZnO}$ nanorod-modified $\mathrm{RGO}$, and the electrocatalytic properties for $\mathrm{H}_{2} \mathrm{O}_{2}$ reduction were examined. ${ }^{63}$ 


\section{Characteristic Features in the Preparations of MNP-RGO}

\subsection{Remarkable variety of preparation methods}

In the present review, we surveyed recent literature with the key words of "graphene" and "nanoparticles", and selected works on electroanalysis. As a result, we found a remarkable variety of preparation methods. This would be due to the fact that there are various reductive routes from GO and metal ions. In Table 1, we summarize the characteristics and performance features of the recent electroanalytical works cited in this review.

While some examples of electrochemical and chemical preparations of MNPs on electrode surfaces were demonstrated in the previous review, ${ }^{1}$ it is recognized that the combination of GO has significantly increased possible preparations. This would be a noticeable difference from the electrode modification of other carbon materials, such as fullerenes ${ }^{64,65}$ and carbon nanotubes. ${ }^{66-68}$ However, the variety of techniques available also suggests that there is no established method for electrode surface modification at present. It seems that each research group adopts their own methodology.

\section{$5 \cdot 2$ GO as the starting material}

While GO has enhanced the variety of the preparations by involving the redox reactions, it is also worthwhile mentioning that the preparation of GO in solutions is now well-established ${ }^{13-15}$ and commonly carried out. Poor solubility of carbon materials would make treatment troublesome, but GO is soluble in water and other organic solvents. Hence, the chemical reactions of GO to form the nanocomposites can be easily carried out. Also, the solution phase preparation is suitable for mass production. Although the reduced form (RGO) might have lower solubility, some treatments are known to make RGO soluble; ${ }^{46,69,70}$ furthermore, if MNP-RGO is formed, it tends to become soluble. Therefore, the treatments in solution phase are inferred to lead to various advances of the MNP-RGO preparations.

As a practical point, the color of the solution is informative for determining the progress of the reduction of GO. Figure 5A shows a photo of an aqueous solution of GO and RGO prepared by reduction with hydrazine,$^{69}$ in $\mathrm{DMF},{ }^{70}$ and by heat treatment. ${ }^{46}$ The color change from brown to blackish is an indicator of the progress of the reduction. The degree of the reduction should change according to the reduction methods as recognized by the changes in absorption spectra as shown in Fig. 5B. In this case, the reduction with hydrazine (curve c) was effective in comparison with that by heat treatment (curve b), suggesting significant recovery of the electronic conjugation of graphene after the hydrazine reduction. While the difference appeared as the shift of the absorption maximum, the conversion of GO to RGO was recognized by the increase in the absorption in the visible region. The effects of the reduction degree are described in the next section.

\subsection{Various degree of the reduction of GO}

Since the conductivity of graphene is much better than that of $\mathrm{GO},{ }^{17}$ a recovery of the conductivity of RGO should be a measure of the progress of the reduction. The higher conductivity of RGO should be beneficial in electrochemical measurements. However, in some reports, even when GO was combined with MNPs without reduction, it seems that the lower conductivity of GO was not a cause of troubles in electrochemical measurements. ${ }^{30,39}$ Therefore, a complete or significant reduction of GO is not essential for preparing the MNP-RGO.

It is inferred that the degree of the reduction of GO would
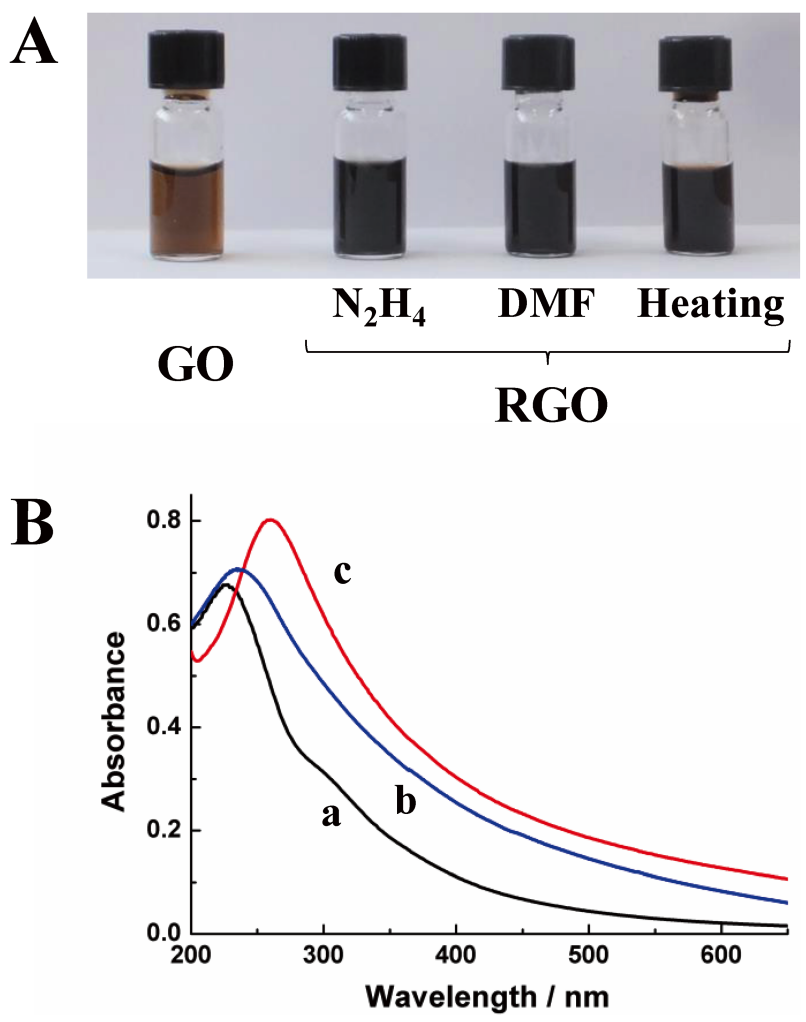

Fig. 5 (A) Photos of the solutions of GO and RGO. The solutions of RGO were prepared by hydrazine reduction, ${ }^{69}$ in $\mathrm{DMF}^{70}$ and by heat treatment. ${ }^{46}$ (B) UV-visible absorption spectra of the solutions of (a) $\mathrm{GO}$ and $(\mathrm{b}, \mathrm{c}) \mathrm{RGO}$. The solutions of RGO were prepared by (b) heat treatment ${ }^{46}$ and (c) hydrazine reduction. ${ }^{69}$

depend on the preparation method, and that some remaining oxygen atoms would not significantly affect the performance of electroanalysis. The degree of the reduction from GO to RGO is not reported explicitly in many cases. However, qualitative progress of the reduction would be recognized from the color change as mentioned in section $5 \cdot 2$.

\subsection{Functions of GO in chemical reactions involving metal ions}

In the chemical reactions of GO or RGO toward metal ions, the multi-functionality of GO should be noted. While the original purpose of GO moieties might be to serve as a support or counterpart for MNPs, GO can work as an anchor of metal ions or MNPs and a kind of capping or stabilizing reagent of MNPs. Therefore, in some cases, "clean" synthesis of MNP$\mathrm{GO}^{30,49}$ or MNP-RGO ${ }^{8-10,45,47,48,71}$ is possible without using special capping reagents. The cleanness, i.e., without using protective organic molecules, should be beneficial for electrochemical and catalytic applications. Furthermore, the synthesis would be suitable for strategically designing bimetallic NP-RGO. ${ }^{9,10,45,71}$ As for some actual formed structures and bimetallic characteristics, Figs. 6 and 7 show the representative TEM and HRTEM images of Pt nanoflower-RGO and Pt-Pd NP-RGO ${ }^{10}$ and the HAADF-STEM characterizations of Pt-Pd nanocube-RGO ${ }^{9}$ and Pt-Pd NP-RGO ${ }^{10}$ with element mappings, respectively.

In the chemical preparations, MNP-RGO can be prepared in solution or isolated as solids, and then modified on the electrode surface in the final step (see Fig. 4). The convenient usability of 

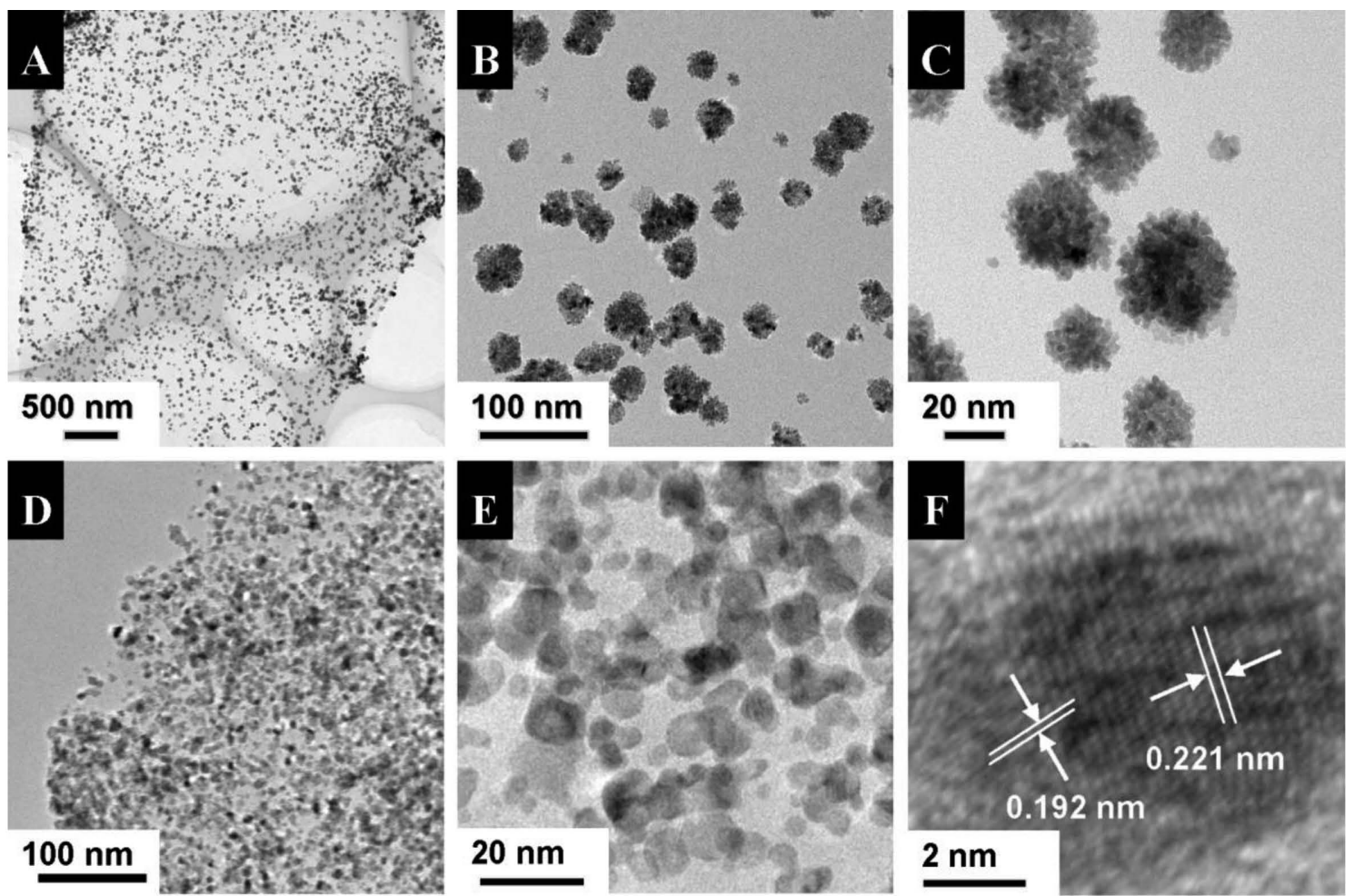

Fig. 6 Representative (A - E) TEM and (F) HRTEM images of (A - C) Pt nanoflower-RGO and (D - F) Pt-Pd NP-RGO. Reproduced from Ref. 10, copyright 2014, with permission from the Royal Society of Chemistry.
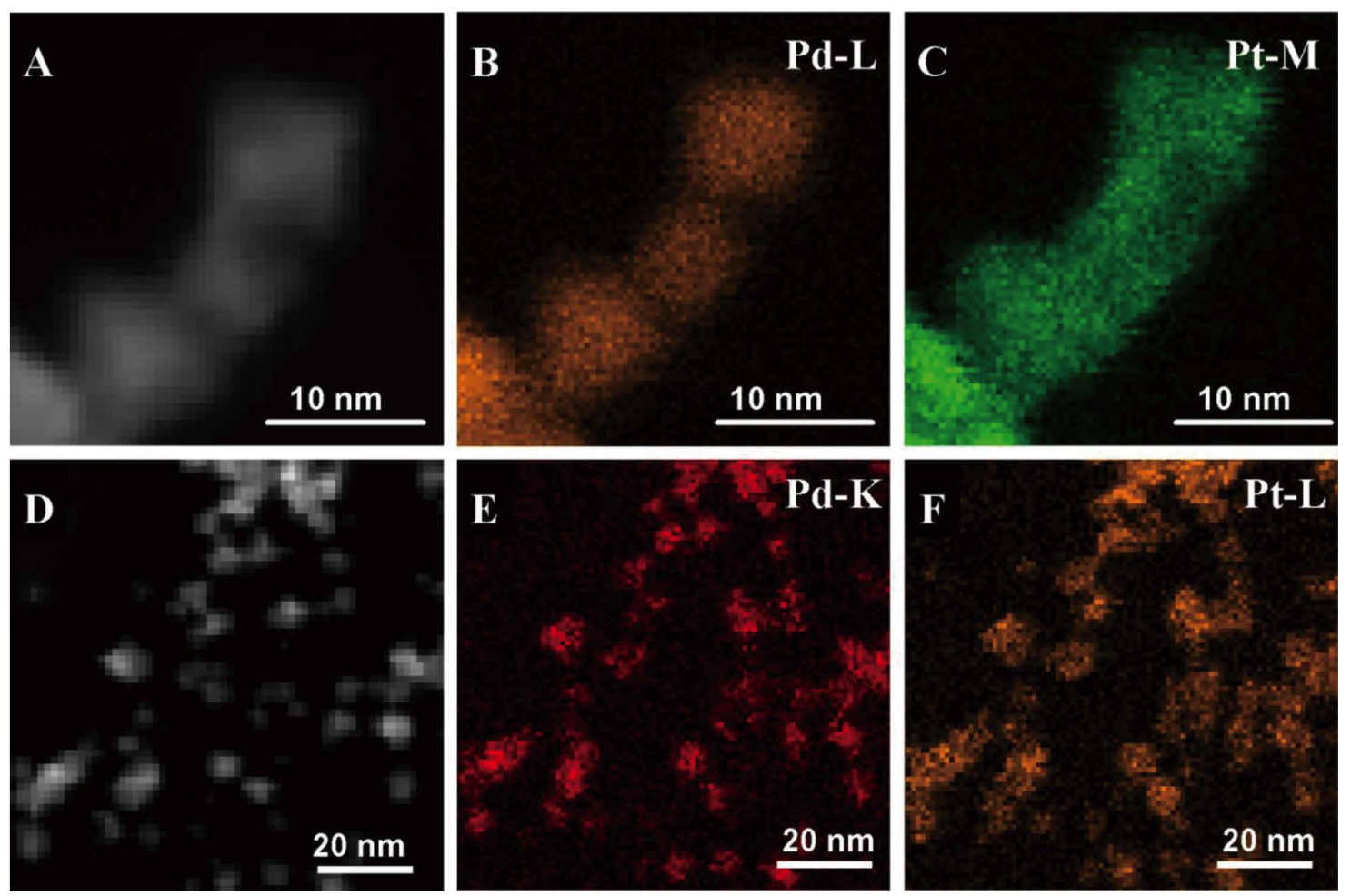

Fig. 7 HAADF-STEM characterizations of (A - C) Pt-Pd nanocube-RGO and (D - F) Pt-Pd NP-RGO. Elemental mapping of (B, E) Pd and (C, F) Pt. Reproduced from Refs. 9 and 10, copyright 2014, with permission from Elsevier and the Royal Society of Chemistry (color version of this figure can be viewed online). 
chemically prepared MNP-RGO as an electrode modifier, for various electrodes just before the electrochemical measurements, is a significant practical merit for preparing modified electrodes for electroanalysis.

\subsection{Variety of graphene derivatives}

Various chemically modified graphenes have been identified in a previous review. ${ }^{16}$ In addition, some functionalized graphenes, such as PDA/RGO, ${ }^{50,51}$ PVP functionalized RGO, ${ }^{52}$ and sulfonated graphene $\mathrm{e}^{54,55}$ can be utilized in preparing the MNP-RGO as mentioned in the previous section. Thus, the variety in functionalizing GO or RGO may lead to some advanced preparations of MNP-RGO. In our very recent work, some differences between citrate-functionalized graphene and PVP-functionalized graphene with AuNPs were explored. ${ }^{72}$

In addition, doped graphenes may increase the variety. Browiec et al. reported the synthesis of $\mathrm{N}$-doped graphene nanosheets decorated with AuNPs for electrochemical determination of chloramphenicol, ${ }^{73}$ though we did not devote much attention to works on doped graphenes in this review.

\section{Concluding Remarks}

In this review, recent nanoarchtectures of MNP-RGO were collected and summarized. Among the notable properties of graphene are its large surface area, excellent electrical conductivity, high thermal conductivity, strong mechanical strength and good biocompatibility. ${ }^{2-5}$ As for the combination with MNPs, the diversity of preparation methods from GO in solutions might be regarded as a practical driving force for promoting the development of MNP-RGO modified electrodes. While in earlier works, preparation of graphene-modified electrodes was performed via the chemical vapor deposition of multilayer graphene nanoflake films ${ }^{74}$ nowadays, wet chemical methods can contribute significantly to the development of MNP-RGO modified electrodes.

The diversity of preparation techniques might imply some complexity. However, it may also indicate greater possibilities for the fabrication of tailor-made electrodes or electrochemical sensors. A variety of analytical targets are summarized in Table 1. Also, comparisons with other modified electrodes have been performed in many cases, and the importance of the MNP-RGO as electrode modifiers have been verified.

While studies in the area of electrochemical preparations are active, we sense that there is considerable potential and possibilities in the field of chemical preparation methods. The pursuit of clean synthesis of MNP-RGO ${ }^{45,47,48}$ should be effective for electroanalysis as well as for application as catalysts in homogeneous solutions. ${ }^{71}$

Despite the volume of research activity on MNP-RGO as summarized in Table 1, the publication of grapheneelectrochemistry studies in Analytical Sciences is very limited. ${ }^{75}$ Thus, we are happy if the present review helps to introduce the interesting properties of graphene derivatives, in particular its use in combination with MNPs in electroanalysis.

\section{Acknowledgements}

Xiaomei Chen thanks the Japan Society for the Promotion of Science (JSPS) for the postdoctoral fellowship. The authors thank JSPS KAKENHI Grant (Nos. 2402335 and 24550100) and the National Nature Scientific Foundation of China (No. 1175112).

\section{References}

1. M. Oyama, Anal. Sci., 2010, 26, 1.

2. S. Guo and S. Dong, J. Mater. Chem., 2011, 21, 18503.

3. X. Chen, G. Wu, Y. Jiang, Y. Wang, and X. Chen, Analyst, 2011, 136, 4631.

4. B. Pérez-Lopéz and A. Merkoçi, Microchim. Acta, 2012, $179,1$.

5. A. Walcarius, S. D. Minteer, J. Wang, Y. Lin, and A. Merkoçi, J. Mater. Chem. B, 2013, 1, 4878.

6. K. Ji, G. Chang, M. Oyama, X. Shang, X. Liu, and Y. He, Electrochim. Acta, 2012, 85, 84.

7. Y. Zhang, H. Shu, G. Chang, K. Ji, M. Oyama, X. Liu, and Y. He, Electrochim. Acta, 2013, 109, 570.

8. G. Wu, H. Huang, X. Chen, Z. Cai, Y. Jiang, and X. Chen, Electrochim. Acta, 2013, 111, 779.

9. X. Chen, Z. Cai, X. Chen, and M. Oyama, Carbon, 2014, 66, 387.

10. X. Chen, Z. Cai, X. Chen, and M. Oyama, J. Mater. Chem. $A, \mathbf{2 0 1 4}, 2,315$.

11. K. S. Novoselov, A. K. Geim, S. V. Morozov, D. Jiang, Y. Zhang, S. V. Dubonos, I. V. Grigorieva, and A. A. Firsov, Science, 2004, 306, 666.

12. A. K. Geim and K. S. Novoselov, Nat. Mater, 2007, 6, 183.

13. W. Hummers and R. Offeman, J. Am. Chem. Soc., 1958, 80, 1339.

14. N. I. Kovtyukhova, P. J. Ollivier, B. R. Martin, T. E. Mallouk, S. A. Chizhik, E. V. Buzaneva, and A. D. Gorchinskiy, Chem. Mater., 1999, 11, 771.

15. L. J. Cote, F. Kim, and J. Huang, J. Am. Chem. Soc., 2008, 131, 1043.

16. S. Park and R. S. Ruoff, Nat. Nanotechnol., 2009, 4, 217.

17. S. Stankovich, D. A. Dikin, R. D. Piner, K. A. Kohlhaas, A. Kleinhammes, Y. Jia, Y. Wu, S. T. Nguyen, and R. S. Ruoff, Carbon, 2007, 45, 1558.

18. S. L. Ting, C. X. Guo, K. C. Leong, D. H. Kim, C. M. Li, and P. Chen, Electrochim. Acta, 2013, 111, 441.

19. Y. Tang, R. Huang, C. Liu, S. Yang, Z. Lu, and S. Luo, Anal. Methods, 2013, 5, 5508.

20. Y. Zhang, Y. Zhao, S. Yuan, H. Wang, and C. He, Sens. Actuators, B, 2013, 185, 602.

21. M. F. Hossain and J. Y. Park, RSC Adv., 2013, 3, 16109.

22. S. Stankovich, D. A. Dikin, G. H. B. Dommett, K. M. Kohlhaas, E. J. Zimney, E. A. Stach, R. D. Piner, S. T. Nguyen, and R. S. Ruoff, Nature, 2006, 442, 282.

23. L. Shang, F. Zhao, and B. Zeng, Electroanalysis, 2013, 25, 453.

24. J. Du, R. Yue, F. Ren, Z. Yao. F. Jiang, P. Yang, and Y. Du, Gold Bull., 2013, 46, 137.

25. Z. Yao, M. Zhu, F. Jiang, Y. Du, C. Wang, and P. Yang, J. Mater. Chem., 2012, 22, 13707.

26. Y. Liu, Z. Huang, Q. Xie, L. Sun, T. Gu, Z. Li, L. Bu, S. Yao, X. Tu, X. Luo, and S. Luo, Sens. Actuators, B, 2013, 188, 894.

27. Z. Lu, S. Yang, Q. Yang, S. Luo, C. Liu, and Y. Tang, Microchim. Acta, 2013, 180, 555.

28. S. Palanisamy, S. Ku, and S. Chen, Microchim. Acta, 2013, 180, 1037.

29. Y. Qin, Y. Kong, Y. Xu, F. Chu, Y. Tao, and S. Li, J. Mater. Chem., 2012, 22, 24821.

30. X. Chen, G. Wu, J. Chen, X. Chen, Z. Xie, and X. Wang, J. Am. Chem. Soc., 2011, 133, 3693.

31. X. Wang, M. Wu, W. Tang, Y. Zhu, L. Wang, Q. Wang, P. He, and Y. Fang, J. Electroanal. Chem., 2013, 695, 10. 
32. X. Ma, Z. Liu, C. Qiu, T. Chen, and H. Ma, Microchim. Acta, 2013, 180, 461.

33. J. Han, Y. Zhuo, Y. Chai, L. Mao, Y. Yuan, and R. Yuan, Talanta, 2011, 85, 130.

34. G. Goncalves, P. A. A. P. Marques, C. M. Granadeiro, H. I. S. Nogueira, M. K. Singh, and J. Gracio, Chem. Mater., 2009, 21, 4796.

35. F. Cui and X. Zhang, J. Electroanal. Chem., 2012, 669, 35.

36. P. Zhang, X. Zhang, S. Zhang, X. Lu, Q. Li, Z. Su, and G. Wei, J. Mater. Chem. B, 2013, 1, 6525.

37. B. Kaur, T. Pandiyan, B. Satpati, and R. Srivastava, Colloids Surf. B, 2013, 111, 97.

38. M. Y. Wang, T. Shen, M. Wang, D. Zhang, and J. Chen, Mater. Lett., 2013, 107, 311.

39. J. Li, D. Kuang, Y. Feng, F. Zhang, Z. Xu, M. Liu, and D. Wang, Biosens. Bioelectron., 2013, 42, 198.

40. C. Xu and X. Wang, Small, 2009, 5, 2212.

41. K. Wu, X. Li, C. Dong, L. Liu, P. Liu, T. Ding, J. Lu, and X. Wei, Chem. Lett., 2013, 42, 1466.

42. J. Yan, S. Liu, Z. Zhang, G. He, P. Zhou, H. Liang, L. Tian, X. Zhou, and H. Jiang, Colloids Surf. B, 2013, 111, 392.

43. W. He, H. Jiang, Y. Zhou, S. Yang, X. Xue, Z. Zou, X. Zhang, D. L. Akins, and H. Yang, Carbon, 2012, 50, 265.

44. L. Gao, W. Yue, S. Tao, and L. Fan, Langmuir, 2013, 29, 957.

45. X. Chen, X. Tian, L. Zhao, Z. Huang, and M. Oyama, Microchim. Acta, in press, DOI: 10.1007/s00604-013-11420 .

46. Z. Lin, Y. Yao, Z. Li, Y. Liu, Z. Li, and C. P. Wong, J. Phys. Chem. C, 2010, 114, 14819.

47. X. Chen, Z. Cai, Z. Huang, M. Oyama, Y. Jiang, and X. Chen, Electrochim. Acta, 2013, 97, 398.

48. X. Chen, Z. Cai, Z. Huang, M. Oyama, Y. Jiang, and X. Chen, Nanoscale, 2013, 5, 5779.

49. X. Chen, B. Su, G. Wu, C. Yang, Z. Zhuang, and X. Chen, J. Mater. Chem., 2012, 22, 11284.

50. Q. Zhang, T. Xu, J. Wei, J. Chen, A. Wang, and J. Feng, Electrochim. Acta, 2013, 112, 127.

51. L. Guo, Q. Liu, G. Li, J. Shi, J. Liu, T. Wang, and G. Jiang, Nanoscale, 2012, 4, 5864.

52. W. Zhu, T. Chen, X. Ma, H. Ma, and S. Chen, Colloids Surf. B, 2013, 111, 321.

53. S. Liu, J. Liu, X. Han, Y. Cui, and W. Wang, Biosens. Bioelectron., 2010, 25, 1640.
54. Y. Si and E. T. Samulski, Nano Lett., 2008, 8, 1679.

55. S. Li, G. Zhao, R. Zhang, Y. Hou, L. Liu, and H. Pang, Microchim. Acta, 2013, 180, 821.

56. D. Deng, S. Li, M. Zhang, X. Liu, M. Zhao, and L. Liu, Anal. Methods, 2013, 5, 2536.

57. F. Xiao, Y. Li, X. Zan, K. Liao, R. Xu, and H. Duan, Adv. Funct. Mater, 2012, 22, 2487.

58. S. Pei, J. Zhao, J. Du, W. Ren, and H. Cheng, Carbon, 2010, 48, 4466.

59. F. Xiao, J. Song, H. Gao, X. Zan, R. Xu, and H. Duan, ACS Nano, 2012, 6, 100.

60. X. Zan, Z. Fang, J. Wu, F. Xiao, F. Huo, and H. Duan, Biosens. Bioelectron., 2013, 49, 71.

61. Q. Zhuo, Y. Ma, J. Gao, P. Zhang, Y. Xia, Y. Tian, X. Sun, J. Zhong, and X. Sun, Inorg. Chem., 2013, 52, 3141.

62. F. Zhang, Z. Wang, Y. Zhang, Z. Zheng, C. Wang, Y. Du, and W. Ye, Int. J. Electrochem. Sci., 2012, 7, 1968.

63. H. Gu, Y. Yang, J. Tian, and G. Shi, ACS Appl. Mater. Interfaces, 2013, 5, 6762 .

64. R. N. Goyal, V. K. Gupta, M. Oyama, and N. Bachheti, Talanta, 2007, 71, 1110.

65. R. N. Goyal, M. Oyama, N. Bachheti, and S. P. Singh, Bioelectrochemistry, 2009, 74, 272.

66. R. N. Goyal, M. Oyama, V. K. Gupta, S. P. Singh, and R. A. Sharma, Sens. Actuators, B, 2008, 134, 816.

67. R. N. Goyal, A. R. S. Rana, M. A. Aziz, and M. Oyama, Anal. Chim. Acta, 2011, 693, 35.

68. R. N. Goyal, S. Bishnoi, H. Chasta, M. A. Aziz, and M. Oyama, Talanta, 2011, 85, 2626.

69. D. Li, M. B. Müller, S. Gilje, R. B. Kaner, and G. G. Wallace, Nat. Nanotechnol., 2008, 3, 101.

70. K. Ai, Y. Liu, L. Lu, X. Cheng, and L. Huo, J. Mater. Chem., 2011, 21, 3365.

71. X. Chen, Z. Cai, X. Chen, and M. Oyama, J. Mater. Chem. $A, \mathbf{2 0 1 4}, 2,5668$.

72. X. Chen, X. Tian, B. Su, Z. Huang, X. Chen, and M. Oyama, Dalton Trans., in press, DOI: 10.1039/c3dt53316f.

73. J. Borowiec, R. Wang, L. Zhu, and J. Zhang, Electrochim. Acta, 2013, 99, 138.

74. N. G. Shang, P. Papakonstantinou, M. McMullan, M. Chu, A. Stamboulis, A. Potenza, S. S. Dhesi, and H. Marchetto, Adv. Funct. Mater, 2008, 18, 3506.

75. Y. Ueno, K. Furukawa, K. Hayashi, M. Takamura, H. Hibino, and E. Tamechika, Anal. Sci., 2013, 29, 55. 